\title{
Transient evolution of quasifree electrons of plasma in liquid water revealed by optical-pump terahertz-probe spectroscopy
}

\author{
Yong Tan, ${ }^{a}$ Hang Zhao, ${ }^{a}$ Rui Zhang, ${ }^{b}$ Yuejin Zhao, ${ }^{a, *}$ Cunlin Zhang, ${ }^{c}$ Xi-Cheng Zhang, ${ }^{d}$ and Liangliang Zhang ${ }^{c, *}$ \\ aBeijing Institute of Technology, School of Optics and Photonics, Beijing Key Laboratory for Precision Optoelectronic Measurement Instruments \\ and Technology, Beijing, China \\ ${ }^{b}$ Chinese Academy of Sciences, Shenzhen Institutes of Advanced Technology, Shenzhen, China \\ 'Capital Normal University, Beijing Advanced Innovation Center for Imaging Technology and Key Laboratory of Terahertz Optoelectronics (MoE), \\ Department of Physics, Beijing, China \\ dUniversity of Rochester, Institute of Optics, Rochester, New York, United States
}

\begin{abstract}
The fundamental properties of laser-induced plasma in liquid water, such as the ultrafast electron migration and solvation, have not yet been clarified. We use 1650-nm femtosecond laser pulses to induce the plasma in a stable free-flowing water film under the strong field ionization mechanism. Moreover, we adopt intense terahertz $(\mathrm{THz})$ pulses to probe the ultrafast temporal evolution of quasifree electrons of the laserinduced plasma in water on the subpicosecond scale. For the first time, the $\mathrm{THz}$ wave absorption signal with a unique two-step decay characteristic in time domain is demonstrated, indicating the significance of electron solvation in water. We employ the Drude model combined with the multilevel intermediate model and particlein-a-box model to simulate and analyze the key information of quasifree electrons, such as the frequencydomain absorption characteristics and solvation ratio. In particular, we observe that the solvation capacity of liquid water decreases with the increase of pumping energy. Up to $~ 50 \%$ of quasifree electrons cannot be captured by traps associated with the bound states as the pumping energy increases to $90 \mu \mathrm{J} / \mathrm{pulse}$. The ultrafast electron evolution in liquid water revealed by the optical-pump/THz-probe experiment provides further insights into the formation and evolution mechanisms of liquid plasma.
\end{abstract}

Keywords: terahertz; liquid water; plasma; solvation.

Received Sep. 23, 2020; revised manuscript received Dec. 1, 2020; accepted for publication Dec. 31, 2020; published online Feb. 3, 2021.

(C) The Authors. Published by SPIE and CLP under a Creative Commons Attribution 4.0 Unported License. Distribution or reproduction of this work in whole or in part requires full attribution of the original publication, including its DOI.

[DOI: 10.1117/1.AP.3.1.015002]

\section{Introduction}

Liquid water is the most common liquid on Earth, and its basic physical and chemical properties are of great significance. ${ }^{1,2}$ In particular, electron migration and solvation associated with photoionization in liquid water is a unique and complex process, which has attracted many researchers to explore various segments of the process. For example, Laenen et al. ${ }^{3}$ used midinfrared femtosecond probe pulses, and Birkedal et al. ${ }^{4}$ employed near-infrared/visible femtosecond probe pulses to observe the latter stage of the electron solvation process. Recently,

*Address all correspondence to Yuejin Zhao, yjzhao @ bit.edu.cn; Liangliang Zhang, liangliang_zhang@cnu.edu.cn
Savolainen et al..$^{5}$ studied the initial stage of this process by adopting terahertz $(\mathrm{THz})$ probe pulses and revealed the initial delocalization length of the excited excess electrons in water. These previous studies constitute a motion picture of an excess electron, which first delocalizes in the conduction band and then is solvated within about 1 to $2 \mathrm{ps}^{{ }^{4-13}}$ This process is considered to include many transient and highly active intermediates, resulting in a large blueshift in the absorption spectrum, from the $\mathrm{THz}$ or gigahertz region to the visible range. Nonetheless, previous studies mainly investigated the behavior of low-density quasifree electrons excited by small pump power density, which is far from enough for further understanding the transient evolution of photoexcited plasma in liquid water. 
Laser-induced plasma has attracted widespread attention due to its diverse physical phenomena. For example, plasma excited in the gaseous environment has been widely investigated and applied in many fields, such as femtosecond pulse compression ${ }^{14-16}$ broadband $\mathrm{THz}$ wave emission, ${ }^{17-20}$ and self-healing of light spot. ${ }^{21}$ However, compared with the numerous studies of plasma in the gaseous environment, the properties of photoexcited plasma in the liquid environment have not been fully revealed, especially in water with large molecular density and strong molecular interactions. Theoretically, laser-induced plasma in liquid water will be accompanied by more complex and stronger nonlinear effects than those in gas, since water has a higher nonlinear coefficient, ${ }^{22}$ a lower excitation threshold, and a higher electron density. ${ }^{23,24}$ In the latest research, the femtosecond laser-induced plasma in water has been used to emit strong $\mathrm{THz}$ waves. ${ }^{25-28}$ In these studies, the physical mechanism of plasma formation in liquid water is considered as an extension of the theory of gas plasma. However, the effect of water molecules on plasma is not fully unraveled. In addition, to the best of our knowledge, there are no reports on the electron migration and solvation process related to strong laser excited plasma in water, especially the initial stage of the interaction between the laser and water on the subpicosecond timescale.

In this paper, we use intense $\mathrm{THz}$ pulses (peak electric field strength of $14.9 \mathrm{MV} / \mathrm{cm}$ ) and a stable free-flowing water film to probe the absorption characteristics of the plasma in liquid water. The femtosecond laser pulses at $1650 \mathrm{~nm}$ are used to excite the plasma in liquid water under the strong field mechanism. We observe that the absorption characteristics of the $\mathrm{THz}$ wave by plasma show a clear two-step decay process in the time domain, which can be explained by the partial quasifree electron solvation. We connect this $\mathrm{THz}$ wave absorption characteristic by plasma with the change of quasifree electron density through the Drude model and combine the multilevel intermediate model with the particle-in-a-box model to analyze the temporal evolution process of quasifree electrons. The absorption changes of $\mathrm{THz}$ waves and proportions of solvated electrons in water under various pumping energies are also revealed.

\section{Experimental Results and Analysis}

The schematic diagram of the optical-pump THz probe spectroscopy system is shown in Fig. 1(a). The intense THz pulses are generated by the organic 4-N,N-dimethylamino-4'-N'-methylstilbazoliumtosylate (DAST) crystal under excitation with the femtosecond laser pulses $(0.4 \mathrm{~mJ}$ per pulse, repetition rate of $1 \mathrm{kHz}$, and center wavelength of $1550 \mathrm{~nm}$ ) from an optical parametric amplifier (OPA, Spectra Physics). To reach the diffraction limited focusing and allow for high field (peak electric field strength of $14.9 \mathrm{MV} / \mathrm{cm}$ ), we use the $\mathrm{THz}$ bullet lambda cubic wave front correction scheme. ${ }^{29}$ The idler-wave output from the OPA is used as pump laser for photoionization (repetition rate of $1 \mathrm{kHz}$ and center wavelength of $1650 \mathrm{~nm}$ ) and focused onto the water film to excite the plasma by a lens with a focal length of 4 in. placed in front of parabolic mirror 1 (PM1). The linearly polarized THz pulses reflected from PM1 and linearly polarized optical pump pulses are transmitted collinearly through the liquid water. In order to efficiently record the evolution process on the subpicosecond time scale, we adjust the relative time delay between the $\mathrm{THz}$ probe and $1650 \mathrm{~nm}$ pump pulse with an accuracy of $10 \mathrm{fs}$. The residual NIR is filtered out using a set of low-pass filters (QMC Instruments Ltd.) with cut-off frequencies of $18 \mathrm{THz}$. The $\mathrm{THz}$ energy is controlled by two $\mathrm{THz}$ polarizers. The $\mathrm{THz}$ pulses passing through the water film are collected by two off-axis parabolic mirrors and collinearly focused with the probe pulses onto the $100-\mu \mathrm{m} \mathrm{GaP}$ crystal for electro-optical sampling. Since the measured broadband $\mathrm{THz}$ pulse will be additionally distorted by the frequency-dependent factors in the electro-optical detection crystal (such as phase matching, reflection, dispersive propagation, and absorption), we use the full complex response function of the $\mathrm{GaP}$ detector to reconstruct all of the spectral information collected in this work. $^{30-32} \mathrm{~A}$ wire-guided, gravity-driven apparatus is employed in this work, which can produce optically stable, flowing thin liquid film and avoid interference caused by a traditional cuvette. ${ }^{33,34}$ Two aluminum wires with diameters of $100 \mu \mathrm{m}$ are separated by $\sim 5 \mathrm{~mm}$ to generate a stable free-flowing water film. The thickness of the water film is $90 \pm 4 \mu \mathrm{m}$ by controlling the water flow rate of $\sim 20 \mathrm{~mL} / \mathrm{min}$. An optical secondharmonic intensity autocorrelator is used to measure and calibrate the thickness. ${ }^{27}$ Within the time interval of $1 \mathrm{~ms}$ between two adjacent pulses, the distance that the gravity-driven water film falls is about $740 \mu \mathrm{m}$, which is much larger than the pump spot size of $\sim 200 \mu \mathrm{m}$. Therefore, the adopted free-flowing water film in our study can effectively avoid the artifacts from cuvette windows and the heat accumulation effect in
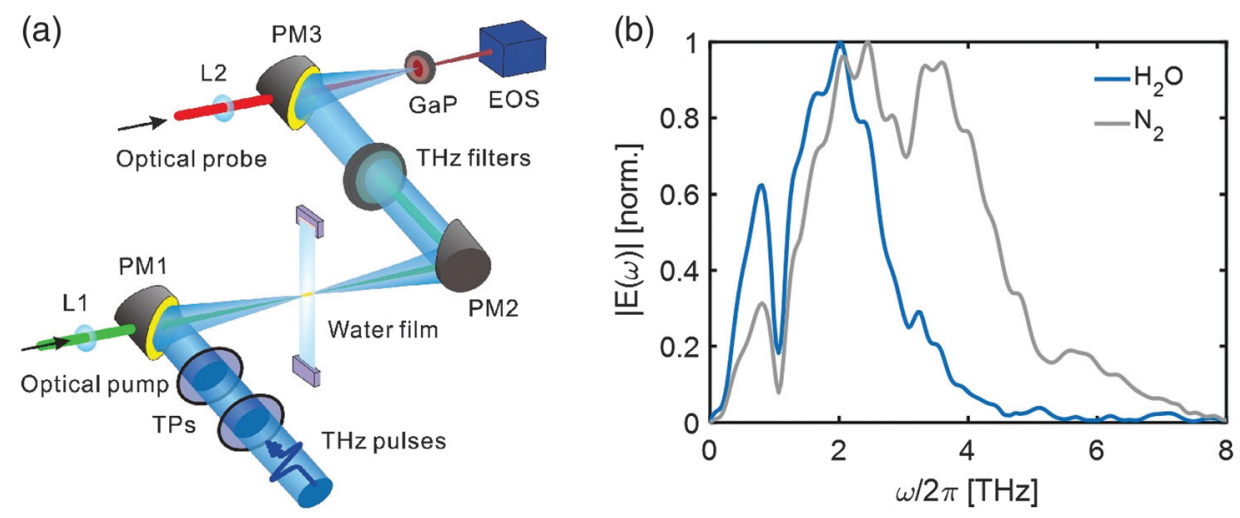

Fig. 1 (a) Diagram of the experimental system. TPs, THz polarizers; PM, parabolic mirror; EOS, electro-optical sampling. (b) The spectrums of $\mathrm{THz}$ pulses propagating through the water film and nitrogen gas (remove the water film) without an optical-pump, respectively. 
the excitation volume. All of the measurements were conducted at the room temperature of $22 \pm 1^{\circ} \mathrm{C}$. For all of the measurements, the system was purged with dry nitrogen gas to improve the signal-to-noise ratio.

Figure 1(b) shows the frequency-domain amplitudes of the $\mathrm{THz}$ electric field pulses propagating through the water film and nitrogen gas with the optical pump beam blocked, and the black curves in Fig. 2(a) (and its inset) show the corresponding time-domain waveforms. Compared with the spectrum in nitrogen gas, the liquid water film has a very strong absorption effect for the high-frequency components of $\mathrm{THz}$ pulses. The $\mathrm{THz}$ spectrum transmitted through water film extends from $\sim 0.2$ to $\sim 4 \mathrm{THz}$ with the peak at $\sim 2.0 \mathrm{THz}$, and there is almost a flat phase for the entire measured spectrum. We fix the $\mathrm{THz}$ scanning time delay $\left(t_{\mathrm{THz}}\right)$ at the peak of the THz pulse and scan the pump-probe time delay $\left(t_{\mathrm{pp}}\right)$ to obtain the transient temporal evolution of the $\mathrm{THz}$ wave absorption change $\left(\Delta E=E_{\text {ref }}-\right.$ $\left.E_{\text {pump }}\right)$ induced by the formed plasma in water on the subpicosecond time scale, as shown in Fig. 2(b). In comparison, the temporal evolution of $\mathrm{THz}$ wave absorption characteristics induced by the formed plasma in nitrogen gas (without the water film) is also presented in Fig. 2(b). The temporal characteristics and corresponding mechanism in the gas phase have been widely investigated. ${ }^{35,36}$ The results of nitrogen gas in our experiment are consistent with previous studies. The experimental evidence in Fig. 2 also shows that in the presence of photoexcited plasma, the THz wave is significantly absorbed in water with a peak modulation more than five times larger than that in nitrogen gas under the same optical pump power. The corresponding time-domain $\mathrm{THz}$ pulses under the maximal absorption by plasma in water and nitrogen gas are shown in Fig. 2(a) and its inset, respectively. Since the absorption of $\mathrm{THz}$ waves by plasma is isotropic, the change of polarization angles between the THz pulse and pump pulse has no effect on our results. Moreover, we find that the $\mathrm{THz}$ wave absorption by plasma in water rises rapidly within a few hundred femtoseconds and then exhibits a unique two-step decay characteristic, which is very different from the monotonic decay characteristic of nitrogen gas. The first step of decay for water is an ultrafast process within the subpicosecond scale, and the following is a relatively slow process with a decay time exceeding over hundreds of picoseconds [as shown in the inset of Fig. 2(b)]. We use a double exponential decay model ${ }^{11}$ to describe the above two-step decay process $\left(A\left[1-A_{1} / \tau_{1} \exp \left(-t / \tau_{1}\right)-A_{2} / \tau_{2} \exp \left(-t / \tau_{2}\right)\right]\right.$, where $A_{1}, A_{2}$ are derived from measured results of solvation rate $\left[A_{1} /\left(A_{1}+A_{2}\right)\right]$, which is closely related to the pump energy; here, $A_{1} \approx 45 \%, A_{2} \approx 55 \%$ for the pump energy of $90 \mu \mathrm{J} /$ pulse). The fitted results, depicted by the orange line in Fig. 2(b) (and its inset), match the experimental data well, and the obtained time constants are $\sim 102$ fs and $\sim 160 \mathrm{ps}$, respectively.

To explain the unique absorption characteristics in liquid water, particles produced or modified by photoionization, including ions, excited neutral molecules, and electrons in bound and quasifree states, need to be considered. The $\mathrm{THz}$ response may be influenced by the movement of electrons or the disturbance of the nuclear spectrum of the disturbed water network. Related to the charged nuclear response, for example, the change in the molar extinction coefficient of water caused by $\mathrm{NaCl}$ dissolution is $1 \times 10^{-2} \mathrm{mM}^{-1} \mathrm{~cm}^{-1} \cdot{ }^{37}$ For the plasma density with the order of $\sim 10^{23} \mathrm{~m}^{-3}$ in this work, the produced charged nuclear response contributes $<0.002 \%$ to the $\mathrm{THz}$ wave absorption change, which is several orders of magnitude smaller than the measured peak modulation magnitude. In addition, the temperature increase due to photoionization in water is also a factor to be considered. In our experiment, the adopted stable free-flowing water film is constantly refreshed, which significantly limits the thermal accumulation. Considering the factors such as the heat capacity and absorption coefficient of liquid water, we estimate that each optical pulse $(\sim 90 \mu \mathrm{J})$ increases the temperature of the excited volume of water film by no more than $0.7^{\circ} \mathrm{C}$, and the corresponding absorption change is $<2 \%$. $^{38}$ However, the above reasons cannot explain the large $\mathrm{THz}$ wave absorption change with maximal value of nearly $50 \%$ in this work. Therefore, we believe that the measured modulation mainly originates from the strong interaction between low effective mass electrons and THz pulses. ${ }^{39}$ In liquid water, the electrons in the plasma induced by the ultrashort laser pulses exist in
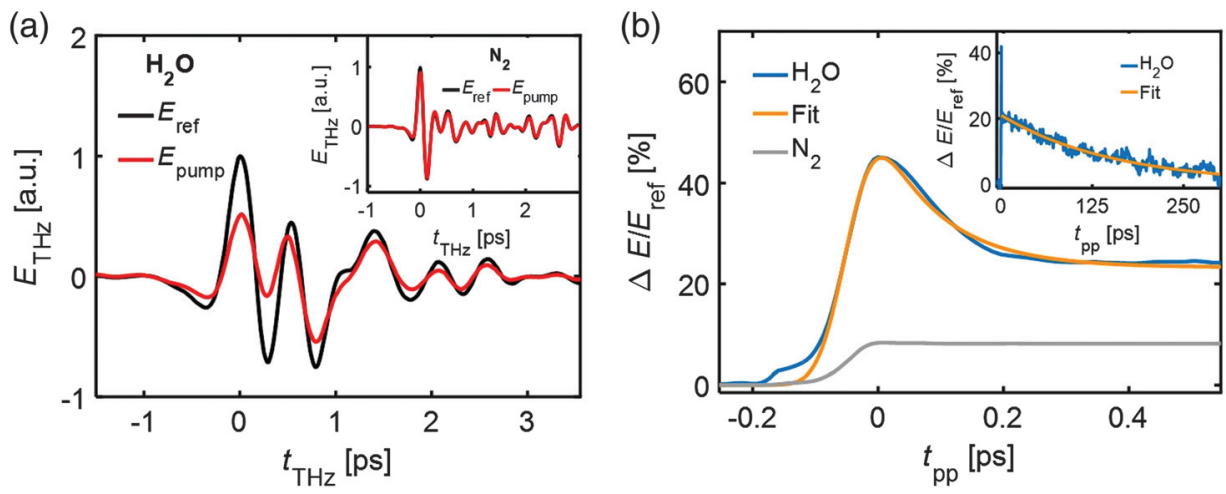

Fig. 2 (a) THz time-domain waveforms in liquid water without an optical pump (black line) and under the maximal absorption caused by the formed plasma (red line); the corresponding results for nitrogen gas are shown in the inset. (b) Transient evolution curve of $\mathrm{THz}$ wave absorption by plasma in water (and nitrogen gas) under a subpicosecond observation window with the pump energy of $90 \mu \mathrm{J} /$ pulse, and the corresponding fitted results for water are shown with the orange line. The inset shows the experimental data and fitted results of the transient evolution process of $\mathrm{THz}$ wave absorption by plasma in water under the observation window of several hundred picoseconds. 
two states, which are solvated electrons and quasifree electrons. The solvated electrons are considered to be trapped in the bound state and do not contribute to the conductivity. They are generated from ionization within about 1 to 2 ps and have a long lifetime ( $\sim$ hundreds of picoseconds) with the characteristic of an optical resonance centered at $720 \mathrm{~nm}$ (bandwidth of about $330 \mathrm{~nm}) .{ }^{5,13,23}$ We do not rule out the weak contribution of solvated electrons to $\mathrm{THz}$ wave absorption, but this obviously cannot explain the dramatic $\mathrm{THz}$ response in the initial stage from no pump beam to the maximal absorption on the subpicosecond scale in this work. Therefore, we mainly attribute the rapid rise of the modulation signal [as shown in Fig. 2(b)] associated with the $\mathrm{THz}$ wave absorption characteristics to the contribution of quasielectrons in the initial stage of plasma formation.

The decay process for quasifree electrons of plasma in liquid water includes geminate recombination, diffusion, and solvation processes. The solvation process dominates on the subpicosecond scale, and the decay time is at least three orders of magnitude faster than other processes. ${ }^{13,40,41}$ Therefore, we believe that the first step of decay in Fig. 2(b) is mainly caused by the solvation of partial quasifree electrons. This also explains the monotonic decay characteristic in nitrogen gas due to the absence of the solvation process. The second-step of decay is considered to be the result of the quasifree electrons being recaptured by the traps associated with the bound states during the demise of hydrated electrons, which mainly reflects the lifetime of hydrated electrons. In general, the formation of hydrated electrons from photoexcited plasma occurs on a time scale of 1 to 2 ps. $^{5,13,23}$ In this work, the modulation signal rapidly decreases with the time constant of $\sim 102$ fs in the first-step of decay. This can be explained by the multilevel intermediate model related to the solvation process. ${ }^{10}$ Specifically, some quasifree electrons transition to the first intermediates with weak $\mathrm{THz}$ wave absorption capacity on a time scale of $\sim 102$ fs. Here, we use the Drude model to evaluate the scattering process for the quasifree electron transitioning into the first intermediate and analyze the $\mathrm{THz}$ wave absorption characteristics based on the quasifree electron density. First, Eq. (1) is adopted to establish the relationship between the alternation of the composite conductivity $[\Delta \sigma(\omega)]$ and the $\mathrm{THz}$ wave absorption change:

$\frac{\Delta E(\omega)}{E_{\mathrm{ref}}(\omega)}=\frac{1}{2 i \omega \varepsilon_{0} n_{2}}\left[\frac{i \omega l}{c}-\frac{n_{2}-n_{1}}{n_{2}\left(n_{2}+n_{1}\right)}+\mathrm{MR}\right] \Delta \sigma(\omega)$,

where $l$ is the thickness of the water film $(l=90 \mu \mathrm{m}), \varepsilon_{0}$ is the dielectric constant in vacuum, and $n_{1}$ and $n_{2}$ are the $\mathrm{THz}$ refractive index in nitrogen gas and liquid water $\left(n_{1} \approx 1\right.$ and $n_{2} \approx 2.07$ ), respectively. $c$ represents the speed of light in vacuum. MR represents the contribution of multiple reflection terms, ${ }^{42}$ which is extremely weak for liquid water with a strong absorption coefficient in the $\mathrm{THz}$ range. Then, the relationship between the induced change in composite conductivity and the quasifree electron density is established, as shown in Eq. (2):

$\Delta \sigma(\omega)=\frac{i \varepsilon_{0} \omega_{p}^{2}}{\omega+i \gamma_{0}}$

where $\omega_{p}=\sqrt{e^{2} n_{f} /\left(\varepsilon_{0} m^{*}\right)}$ represents the plasma frequency, $e$ represents elementary charge $\left(e \approx 1.6 \times 10^{-19} \mathrm{C}\right), m^{*}$ denotes the effective mass of the electron $\left(m^{*} \approx 9.1 \times 10^{-31} \mathrm{~kg}\right), n_{f}$ represents the quasifree electron density, and $\gamma_{0}=\tau_{f}^{-1}$ denotes

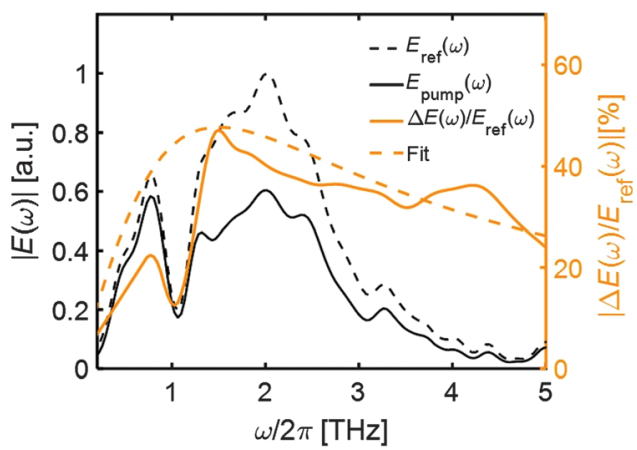

Fig. 3 Experimental (orange solid line) and simulated (orange dotted line) frequency-domain characteristics of $\mathrm{THz}$ wave absorption under the maximal modulation by plasma in water. The black solid line and the black dotted line show the spectra of $\mathrm{THz}$ pulses with the $90-\mu \mathrm{J}$ pump pulse (under the maximal absorption) and without pump pulse, respectively.

the quasifree electron scattering rate, which is directly related to the lifetime of quasifree electrons $\left(\tau_{f} \approx 106 \mathrm{fs}\right)$. Combining Eqs. (1) and (2), it can be concluded that the quasifree electron density in the plasma is proportional to the amplitude change of the $\mathrm{THz}$ electric field.

The experimental frequency-domain characteristics of $\mathrm{THz}$ wave absorption $[\Delta E(\omega) / E(\omega)]$ under maximal modulation by plasma in liquid water are shown in Fig. 3. The simulated results based on the above Drude model are also shown as the orange dotted line. An obvious absorption peak near $\sim 1.5 \mathrm{THz}$ is demonstrated. The simulation results are basically consistent with the experimental data. Note that the measured absorption is slightly lower than the simulated value in the low-frequency range. This is mainly because the $\mathrm{THz}$ pulse tail carrying low-frequency components always lags behind the main pulse $\left(t_{\mathrm{THz}}=0\right)$ to pass through the plasma, and then the quasifree electron density has been partially attenuated. In addition, the absorption dip at $\sim 1 \mathrm{THz}$ in the measurement is mainly derived from the characteristics of the DAST source itself. According to the Drude model, the lifetime of quasifree electrons is calculated to be $\sim 106 \mathrm{fs}$, which is approximately in agreement with the experimental measurement of $\sim 102$ fs. In addition, the calculated maximum value of the quasifree electron density of the plasma excited in liquid water is $\sim 4.5 \times 10^{22} \mathrm{~m}^{-3}$ with the adopted pump energy of $90 \mu \mathrm{J} /$ pulse.

Furthermore, we measure the transient evolution processes of $\mathrm{THz}$ wave absorption by plasma in water with the excitation of different pumping energies and calculate the corresponding modulation degree at $1.5 \mathrm{THz}$ under the maximal absorption $[\Delta E(1.5 \mathrm{THz}) / E(1.5 \mathrm{THz})]$. The results are shown in Fig. 4. We find that the laser-excited peak quasifree electron density has a linear relationship with the pumping energy in the range from 40 to $90 \mu \mathrm{J} /$ pulse, which can be explained by the mechanism of the strong field ionization. ${ }^{43}$ In our experiment, the peak quasifree electron density tends to saturate when the pumping energy is higher than $90 \mu \mathrm{J} /$ pulse.

As the pumping energy increases, the plateau feature after the first step of decay shows an upward trend, as shown in the inset of Fig. 4(a). This is due to the decrease in the proportion of quasifree electrons that are solvated with the increase of pumping energy. For the excited low-density quasifree electrons (such as the condition of the pumping energy $<50 \mu \mathrm{J} /$ pulse), the ratio 

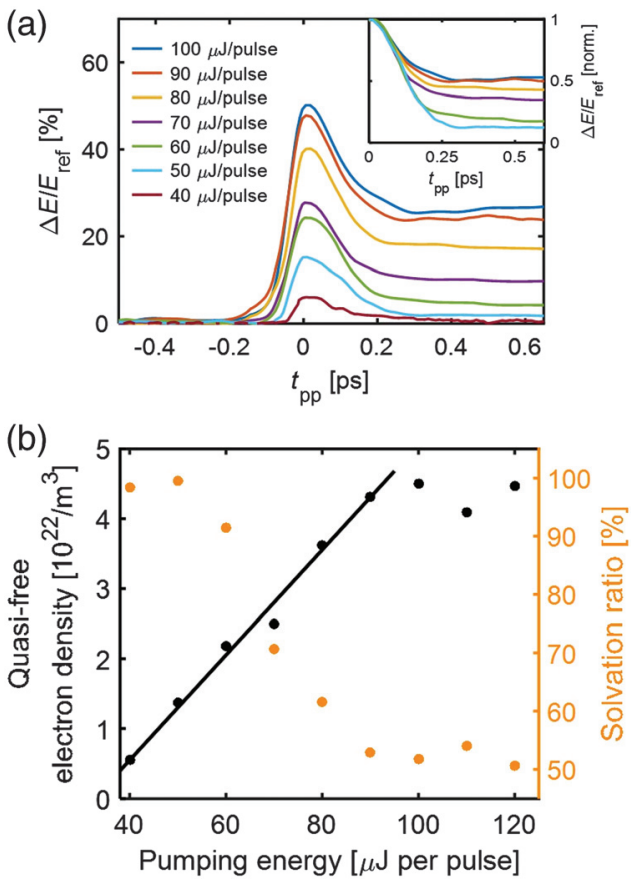

Fig. 4 (a) Transient evolution curves of $\mathrm{THz}$ wave absorption by plasma in water induced by different pump pulse energies. The inset shows the corresponding normalized results from 0 ps. (b) The black points indicate the peak quasifree electron density with different pump pulse energies. The black line is the linear fitting results. The orange points show the relationship between the solvation ratio and the pump pulse energy at the equilibrium state.

of the quasifree electron density $\left(n_{f}\right)$ to the solvated electron density $\left(n_{d}\right)$ at the equilibrium state depends on the relative mobilities, i.e., $n_{f} / n_{d}=\mu_{d} / \mu_{f}$. $\mu_{d}$ and $\mu_{f}$ represent the mobility of solvated electrons and quasifree electrons, respectively. The electron mobility is related to the lifetime $\tau$ of the electron $\left(\mu=e \tau / m^{*}\right)$. Therefore, for quasifree electrons with a lifetime of $\sim 102$ fs and solvated electrons with a lifetime of $\sim 160 \mathrm{ps}$, more than $99.8 \%$ of the quasifree electrons will be solvated at the equilibrium state. We believe that the low-density quasifree electrons will be completely solvated after $\sim 200 \mathrm{fs}$. However, the energy-dependent experimental data of Fig. 4(a) show that the modulation degree in the plateau region is about $0.8 \%$ at the pumping energy of $40 \mu \mathrm{J} /$ pulse, which comes from thermal effects. According to Ref. 38, the modulation degree of $0.8 \%$ is caused by a temperature difference of $\sim 0.29^{\circ} \mathrm{C}$, which is basically consistent with the temperature estimation in our present setup. In addition, the temperature response will not be significant as the pumping energy increases. As shown in Fig. 4(a), as the pumping energy increases from 40 to $90 \mu \mathrm{J} /$ pulse, the modulation degree at the plateau stage increases significantly from $\sim 0.8 \%$ to $\sim 25 \%$. However, even the thermal effect induced by $90 \mu \mathrm{J} /$ pulse $\left(\sim 0.65^{\circ} \mathrm{C}\right)$ only causes a modulation degree of $2 \%$ in $\mathrm{THz}$ absorption. Therefore, considering the increase of the quasifree electron density in the plasma and the factor that the plasma will destroy the network structure of liquid water, we attribute the increase of the modulation degree at the plateau stage with increasing pump pulse energy [the inset of Fig. 4(a)] mainly to the saturation of the traps associated with the bound states. When the traps are saturated, more quasifree electrons cannot be relaxed through the solvation process. Excluding the thermal effect, we calculate the ratio of quasifree electrons solvated with different pumping energies [i.e., solvation rate $A_{1} /\left(A_{1}+A_{2}\right)$, as shown in Fig. 4(b)]. Specifically, the low-density quasifree electrons are almost all captured by the traps related to the bound state with the weak pump pulse excitation $(<50 \mu \mathrm{J} /$ pulse $)$. However, the traps gradually tend to be saturated, and the solvation ratio decreases as the pump pulse energy exceeds the threshold ( $\sim 60 \mu \mathrm{J} /$ pulse in this work). When the quasifree electron density in plasma is saturated, the ratio of quasifree electrons solvated drops to $\sim 50 \%$ with pumping energy higher than $90 \mu \mathrm{J} /$ pulse.

The size of the electron in the plasma after photoionization is also important information and can be regarded as the radius of gyration of the spin density. The particle-in-a-box model is adopted in our work, which describes the solvation of electrons as a continuously changing process. ${ }^{5,44}$ At the initial stage of plasma formation, electrons usually have a large radius of gyration, which is basically equal to their initial average ejection length. The electrons at this time can be regarded as quasifree electrons in an unbound state. Then, the water molecules solvate the electrons and form a cage in a bound state. The electron's gyration radius gradually decreases until the quasifree electrons are solvated within a time scale of 1 to 2 ps. The relationship between the resonance absorption frequency $(f)$ of electrons and the radius of gyration $\left(r_{g}\right)$ can be expressed as

$f=a / h r_{g}^{2}$,

where $h$ is Planck constant, and $a$ is a constant related to the first excitation energy and is about $10.5 \mathrm{eV}^{2}$ in liquid water. ${ }^{5,44}$ Based on Eq. (3), we calculate the initial radius of gyration of quasifree electrons in liquid water at an absorption peak of $1.5 \mathrm{THz}$ as $\sim 40 \AA$ in our experiment, which is in agreement with the previously published results. ${ }^{5}$ As the radius of gyration continuously reduces by the restraint of the water molecule cage, the resonant absorption frequency continues to blueshift and leaves the $\mathrm{THz}$ observation window after $\sim 100 \mathrm{fs}$. Eventually, the resonant absorption frequency converges to the near visible spectrum at around $720 \mathrm{~nm}$ with the corresponding radius of gyration of about $2.5 \AA^{5}$

\section{Conclusion}

$\mathrm{THz}$ time-domain spectroscopy can provide instantaneous information about the frequency-dependent conductivity of the medium excited by optical pump pulses. In this work, we explore the optical-pump/THz-probe experiment to investigate the transient dynamic evolution of quasifree electrons of plasma in water on the subpicosecond time scale. The time-resolved transient $\mathrm{THz}$ wave absorption measurements prove that the quasifree electrons in the laser-excited plasma in water will be solvated rapidly. The measured temporal transmittance data contain valuable information, which can be supported by a combination of the Drude model, the multilevel intermediate model, and the particle-in-a-box model. We conclude that the initial delocalization radius of gyration of quasifree electrons is $\sim 40 \AA$, the scattering time is $\sim 102 \mathrm{fs}$, and the residence time of electrons in the bound state is up to $\sim 160 \mathrm{ps}$. The peak quasifree electron density increases linearly, while the pumping energy increases from 40 to $90 \mu \mathrm{J} /$ pulse and tends to saturate with a value of $\sim 4.5 \times 10^{22} \mathrm{~m}^{-3}$. In addition, we observe that as 
the quasifree electron density increases, the traps related to the bound states appear to saturate, resulting in a large number of quasifree electrons not being completely solvated. When the pump energy increases to $90 \mu \mathrm{J} /$ pulse, the proportion of solvated electrons gradually decreases to about $\sim 50 \%$. This work can provide valuable insights on the fundamental aspects of the charge transport process in water and lay the foundation for further understanding the physicochemical properties and transient evolution of the femtosecond laser pulse excited plasma in water. We believe that the proposed method can also be applied to study the physical mechanisms of plasma deformation for the ionic aqueous solution and other liquids.

\section{Acknowledgments}

This work was supported by the Beijing Natural Science Foundation (Grant No. JQ18015), the National Natural Science Foundation of China (Grant Nos. 61935001, 61905271, 12074272), the Guangdong Basic and Applied Basic Research Foundation (Grant No. 2020A1515011083), and the Beijing Advanced Innovation Center for Imaging Theory and Technology. The authors declare no conflicts of interest.

\section{References}

1. M. C. Bellissent-Funel et al., "Water determines the structure and dynamics of proteins," Chem. Rev. 116(13), 7673-7697 (2016).

2. M. Heyden and D. J. Tobias, "Spatial dependence of protein-water collective hydrogen-bond dynamics," Phys. Rev. Lett. 111(21), 218101 (2013).

3. R. Laenen, T. Roth, and A. Laubereau, "Novel precursors of solvated electrons in water: evidence for a charge transfer process," Phys. Rev. Lett. 85(1), 50-53 (2000).

4. V. Birkedal et al., "Observation of a persistent infrared absorption following two photon ionization of liquid water," Chem. Phys. 328(1-3), 119-124 (2006).

5. J. Savolainen et al., "Direct observation of the collapse of the delocalized excess electron in water," Nat. Chem. 6(8), 697-701 (2014).

6. F. Perakis et al., "Vibrational spectroscopy and dynamics of water," Chem. Rev. 116(13), 7590-7607 (2016).

7. R. M. Young and D. M. Neumark, "Dynamics of solvated electrons in clusters," Chem. Rev. 112(11), 5553-5577 (2012).

8. V. H. Vilchiz et al., "Map for the relaxation dynamics of hot photoelectrons injected into liquid water via anion threshold photodetachment and above threshold solvent ionization," J. Phys. Chem. A 105(10), 1711-1723 (2001).

9. E. Knoesel et al., "Charge transport and carrier dynamics in liquids probed by THz time-domain spectroscopy," Phys. Rev. Lett. 86(2), 340-343 (2001).

10. C. Pepin et al., "Observation of a continuous spectral shift in the solvation kinetics of electrons in neat liquid deuterated water," J. Phys. Chem. A 101(24), 4351-4360 (1997).

11. A. Migus, Y. Gauduel, and J. L. Martin, "Excess electrons in liquid water: first evidence of a prehydrated state with femtosecond lifetime," Phys. Rev. Lett. 58(15), 1559-1562 (1987).

12. H. Iglev et al., "Hydrogen atom transfer from water or alcohols activated by presolvated electrons," J. Phys. Chem. Lett. 6(6), 986-992 (2015).

13. R. Lian, R. A. Crowell, and I. A. Shkrob, "Solvation and thermalization of electrons generated by above-the-gap $(12.4 \mathrm{eV})$ twophoton ionization of liquid $\mathrm{H}_{2} \mathrm{O}$ and $\mathrm{D}_{2} \mathrm{O}$," J. Phys. Chem. A 109(8), 1510-1520 (2005).

14. S. Champeaux and L. Bergé, "Femtosecond pulse compression in pressure-gas cells filled with argon," Phys. Rev. E 68(6), 066603 (2003).
15. S. A. Skobelev, A. V. Kim, and O. Willi, "Generation of highenergy few-cycle laser pulses by using the ionization-induced self-compression effect," Phys. Rev. Lett. 108(12), 123904 (2012).

16. G. Stibenz, N. Zhavoronkov, and G. Steinmeyer, "Self-compression of millijoule pulses to 7.8 fs duration in a white-light filament," Opt. Lett. 31(2), 274-276 (2006).

17. K. Y. Kim, J. H. Glownia, and A. J. Taylor, "Terahertz emission from ultrafast ionizing air in symmetry-broken laser fields," Opt. Express 15(8), 4577-4584 (2007).

18. T. Wu et al., "Excitation-wavelength-dependent terahertz wave modulation via preformed air plasma," Appl. Phys. Lett. 112(17), 171106 (2018).

19. A. Gorodetsky et al., "Physics of the conical broadband terahertz emission from two-color laser-induced plasma filaments," Phys. Rev. A 89(3), 033838 (2014).

20. L. Zhang et al., "Observation of terahertz radiation via the twocolor laser scheme with uncommon frequency ratios," Phys. Rev. Lett. 119(23), 235001 (2017).

21. M. Kolesik and J. V. Moloney, "Self-healing femtosecond light filaments," Opt. Lett. 29(6), 590-592 (2004).

22. R. W. Boyd, Nonlinear Optics, 2nd ed., Elsevier, Amsterdam (2003).

23. S. Minardi et al., "Time-resolved refractive index and absorption mapping of light-plasma filaments in water," Opt. Lett. 33(1), 86-88 (2008).

24. A. Vogel, S. Busch, and U. Parlitz, "Shock wave emission and cavitation bubble generation by picosecond and nanosecond optical breakdown in water," J. Acoust. Soc. Am. 100(1), 148-165 (1996).

25. L. Zhang et al., "Strong terahertz radiation from a liquid-water line," Phys. Rev. Appl. 12(1), 014005 (2019).

26. E. Yiwen et al., "Terahertz wave generation from liquid water films via laser-induced breakdown," Appl. Phys. Lett. 113(18), 181103 (2018).

27. Q. Jin et al., "Observation of broadband terahertz wave generation from liquid water," Appl. Phys. Lett. 111(7), 071103 (2017).

28. I. Dey et al., "Highly efficient broadband terahertz generation from ultrashort laser filamentation in liquids," Nat. Commun. 8(1), 1184 (2017).

29. M. Shalaby and C. P. Hauri, "Demonstration of a low-frequency three-dimensional terahertz bullet with extreme brightness," Nat. Commun. 6(1), 5976 (2015).

30. A. Leitenstorfer et al., "Detectors and sources for ultrabroadband electro-optic sampling: experiment and theory," Appl. Phys. Lett. 74(11), 1516-1518 (1999).

31. Q. Wu and X. C. Zhang, "Free-space electro-optics sampling of mid-infrared pulses," Appl. Phys. Lett. 71(10), 1285-1286 (1997).

32. H. Zhao et al., "Ultrafast hydrogen bond dynamics of liquid water revealed by terahertz-induced transient birefringence," Light Sci. Appl. 9(1), 136 (2020).

33. M. J. Tauber et al., "Flowing liquid sample jet for resonance Raman and ultrafast optical spectroscopy," Rev. Sci. Instrum. 74(11), 4958-4960 (2003).

34. T. Wang, P. Klarskov, and P. U. Jepsen, "Ultrabroadband THz time-domain spectroscopy of a free-flowing water film," IEEE Trans. Terahertz Sci. Technol. 4(4), 425-431 (2014).

35. J. Das and M. Yamaguchi, "Terahertz wave excitation from preexisting air plasma," J. Opt. Soc. Am. B 30(6), 1595-1600 (2013).

36. J. Zhao et al., "Wavelength scaling of terahertz wave absorption via preformed air plasma," IEEE Trans. Terahertz Sci. Technol. 6(6), 846-850 (2016).

37. J. Xu et al., " $0.15-3.72 \mathrm{THz}$ absorption of aqueous salts and saline solutions," Appl. Phys. Lett. 90(3), 031908 (2007).

38. H. R. Zelsmann, "Temperature dependence of the optical constants for liquid $\mathrm{H}_{2} \mathrm{O}$ and $\mathrm{D}_{2} \mathrm{O}$ in the far IR region," J. Mol. Struct. 350(2), 95-114 (1995).

39. L. D. Siebbeles et al., "A subpicosecond pump-probe laser study of ionization and geminate charge recombination kinetics in alkane liquids," J. Chem. Phys. 107(22), 9339-9347 (1997). 
40. J. Noack and A. Vogel, "Laser-induced plasma formation in water at nanosecond to femtosecond time scales: calculation of thresholds, absorption coefficients, and energy density," IEEE J. Quantum Electron. 35(8), 1156-1167 (1999).

41. F. Docchio, "Lifetimes of plasmas induced in liquids and ocular media by single Nd:YAG laser pulses of different duration," Europhys. Lett. 6(5), 407-412 (1988).

42. E. Knoesel et al., "Conductivity of solvated electrons in hexane investigated with terahertz time-domain spectroscopy," J. Chem. Phys. 121(1), 394-404 (2004).

43. Z. Mics, F. Kadlec, and P. Kužel, "Nonresonant ionization of oxygen molecules by femtosecond pulses: plasma dynamics studied by time-resolved terahertz spectroscopy," J. Chem. Phys. 123(10), 104310 (2005).

44. L. D. Jacobson and J. M. Herbert, "Theoretical characterization of four distinct isomer types in hydrated-electron clusters, and proposed assignments for photoelectron spectra of water cluster anions," J. Am. Chem. Soc. 133(49), 19889-19899 (2011).

Yong Tan received his BS degree from Chang'an University, Xi'an, China, in 2016. He is currently pursuing his $\mathrm{PhD}$ in instrument science and technology at Beijing Institute of Technology, Beijing, China. His research interests include instrument science and technology and terahertz emission. His research primarily concerns generation and detection of terahertz waves.

Hang Zhao received her BS degree from Shenyang University of technology, Shenyang, China, in 2014. She received her MS degree from Capital Normal University, Beijing, China, in 2017. She is currently pursuing her PhD in instrument science and technology at Beijing Institute of Technology, Beijing, China. Her research primarily concerns generation and detection of terahertz waves.

Rui Zhang received his BEng degree in electronic information engineering from Xidian University, Xi'an, China, in 2010. He received his PhD in mechanics from Peking University, Beijing, China, in 2016. His research interests include terahertz spectroscopy and imaging, biomedical application of terahertz techniques, and terahertz wave generation. $\mathrm{He}$ is now an associate professor at Shenzhen Institute of Advanced Technology.

Yuejin Zhao received his $\mathrm{PhD}$ in optical engineering from Beijing Institute of Technology, Beijing, China, in 1990. He is currently a professor with the School of Optoelectronics, Beijing Institute of Technology. He has disciplinary responsibility as a team leader of "Instrument Science and Technology" with Beijing Institute of Technology. His current research interests include terahertz imaging technology based on MEMS-infrared imaging technology, space-optical technology, and intelligent photoelectric instrument development.

Cunlin Zhang received his $\mathrm{PhD}$ in photoelectric engineering and optical engineering from Beijing Institute of Technology, Beijing, China, in 1992. $\mathrm{He}$ is currently a professor with the Capital Normal University and also the director of Beijing Key Laboratory of Terahertz Spectrum and Imaging. $\mathrm{He}$ has disciplinary responsibility as a team leader of "Terahertz Spectrum and Imaging" with Beijing Institute of Technology. His current research interests include terahertz and infrared spectrum and imaging.

Xi-Cheng Zhang is the Parker Givens Chair and former director of the Institute of Optics, University of Rochester (UR), New York, USA. With his BS degree in 1982 from Peking University, he received his MS and $\mathrm{PhD}$ degrees in physics from Brown University, Rhode Island, in 1983 and 1986, respectively. He has been recognized with many honors and awards, including the Humboldt Prize, from the Alexander von Humboldt Foundation (2018); IRMMW-THz Kenneth Button Prize (2014); OSA William F. Meggers Award (2012); IEEE Photonics Society William Streifer Scientific Achievement Award (2011); and the Rensselaer William H. Wiley 1866 Award (2009), among others.

Liangliang Zhang received her PhD in instrument science and technology from Beijing Institute of Technology, Beijing, China, in 2008. She is currently a professor with the Department of Physics, Capital Normal University. Her research interests include a wide variety of topics in the area of terahertz emission and terahertz detection. 